\title{
EQUAÇÕES PARA ESTIMAR A COMPOSIÇÃO QUÍMICA E O PODER CALORÍFICO DE BIOMASSAS TRATADAS TERMICAMENTE A PARTIR DA ANÁLISE COLORIMÉTRICA
}

\footnotetext{
Juliana Marangon Jardim¹; Angélica de Cássia Oliveira Carneiro²; Emylle Veloso

Santos Costa ${ }^{3}$; Mateus Alves de Magalhães ${ }^{4}$ Carlos Miguel Simões da Silva ${ }^{5}$

${ }^{1}$ Universidade Federal de Viçosa, estudante de mestrado em Tecnologia de Celulose e Papel, Viçosa, Minas Gerais, Brasil. E-mail: mjardim.juliana@gmail.com.

${ }^{2}$ Universidade Federal de Viçosa, professora do departamento de Engenharia Florestal, Viçosa, Minas Gerais, Brasil.

${ }^{3}$ Universidade Federal de Lavras, estudante de mestrado em Tecnologia da Madeira, Lavras, Minas Gerais, Brasil.

${ }^{4,5}$ Universidade Federal de Viçosa, estudante de doutorado em Ciência Florestal, Viçosa, Minas Gerais, Brasil.
}

Recebido em: 08/04/2016 - Aprovado em: 30/05/2016 - Publicado em: 20/06/2016 DOI: 10.18677/Enciclopedia_Biosfera_2016_146

\begin{abstract}
RESUMO
A biomassa termicamente tratada apresenta um incremento nas suas propriedades de interesse energético, como maiores teores de carbono e energia e menores teores de materiais voláteis e umidade. Todavia, a quantificação desses índices de qualidade é relativamente demorada e de alto custo, o que impulsiona a busca por metodologias alternativas, pelo setor industrial, para a determinação das mesmas. Com isso, o objetivo do presente estudo foi avaliar e propor uma metodologia para se estimar a composição química e o poder calorífico, a partir dos índices colorimétricos, de biomassas submetidas a tratamento térmico. Para a realização do experimento três biomassas (serragem de eucalipto, bagaço de cana-de-açúcar e bambu) foram termicamente tratadas e avaliadas quanto as suas propriedades energéticas. Em seguida procedeu-se análise colorimétrica de acordo com o sistema CIE L*a*b* que trabalha com três índices de cor: claridade (L); coordenadas cromáticas do Matiz Vermelho $\left(a^{*}\right)$ e do Matiz Amarelo $\left(b^{*}\right)$. Verificou-se que o aumento na temperatura do tratamento proporcionou uma redução nesses índices colorimétricos, independente da biomassa. Houve interação significativa entre todos os índices colorimétricos, a composição química imediata e o poder calorífico. As equações de regressão ajustadas para estimar as propriedades energéticas a partir dos índices do colorimetria apresentaram coeficiente de determinação satisfatório, sendo assim, a metodologia proposta apresentou-se viável e com potencial de aplicação.
\end{abstract}

PALAVRAS-CHAVE: Metodologia de Estimação, Sistema CIE L*a* b*, propriedades energéticas. 


\title{
EQUATIONS TO ESTIMATE OF CHEMICAL COMPOSITION AND HEATING VALUE OF THERMAL TREATED BIOMASS FROM COLORIMETRIC ANALYSIS
}

\begin{abstract}
The thermally treated biomass has an increase in its properties of energy interest, as higher levels of carbon and energy and lower levels of volatiles and moisture. However, quantification of these quality indexes is relatively time consuming and expensive, making up with the industrial sector seeks alternative methods for determining the same. Thus, the aim of this study was to evaluate and propose an estimation methodology of the chemical composition and higher heating value of biomass subjected to thermal treatment from their colorimetric indexes. For the experiment three biomasses (eucalyptus sawdust, sugarcane bagasse and bamboo) were thermal treated and evaluated for its energy properties. Then he proceeded to colorimetric analysis according to the $\mathrm{CIE} \mathrm{L}^{*} \mathrm{a}{ }^{*} \mathrm{~b}$ * color that has three indexes: $\mathrm{L}$ - lightness, $\mathrm{a}^{*}$ - coordinate (red coordinate) and $\mathrm{b}^{*}$ - coordinate (yellow coordinate). It was found that the increase in treatment temperature provided a reduction in these colorimetric indexes, independently of biomass. There was a significant interaction between all colorimetric indexes, chemical composition and higher heating value. The regression equations adjusted to estimate the energy properties from the colorimetric indexes showed satisfactory coefficient of determination, therefore, the proposed methodology presented is feasible and with application potential.
\end{abstract}

KEYWORDS: Estimation Methodology, CIE L*a*b*, Energy Properties.

\section{INTRODUÇÃO}

A biomassa, na forma bruta, apresenta uma série de desvantagens como o alto teor de umidade e a baixa densidade energética que dificultam o seu uso direto como combustível e reduzem sua competividade dentro do mercado de energia (ARIAS et al., 2008). Os tratamentos térmicos, como a torrefação (de 200 a $300{ }^{\circ} \mathrm{C}$ ) e a carbonização (acima de $300^{\circ} \mathrm{C}$ ), são algumas das te cnologias empregadas para contornar esses inconvenientes e melhorar os índices de qualidade dessa importante fonte energética (VAN DER STELT et al., 2011). O material resultante apresenta-se com maior poder calorífico, maior teor de carbono, menor teor de materiais voláteis, menor umidade e higroscopicidade reduzida, além da coloração mais escura (ESTEVES \& PEREIRA, 2009; VAN DER STELT et al., 2011; PEREIRA et al., 2013).

O poder calorífico e a composição química imediata (carbono fixo, materiais voláteis e cinzas) são algumas das propriedades mais relevantes para o controle de qualidade e caracterização da biomassa termicamente tratada para produção de energia (OLIVEIRA et al., 2013). Entretanto, essas análises são relativamente caras, exigindo um dispêndio de tempo, mão-de-obra especializada e capital para compra de equipamentos e para a realização das análises em si. No setor industrial, a grande quantidade de amostras e análises e a rapidez exigida faz aumentar a demanda por metodologias alternativas que possam fornecer esses resultados de maneira cada vez mais rápida e barata.

Nesse contexto, o uso de equações para estimar essas propriedades se torna atrativo do ponto de vista econômico e operacional, desde que se possam estimar de maneira fácil e confiável as informações desejadas. Como muitas das propriedades da biomassa apresentam correlações significativas entre si, podem-se gerar equações a partir de variáveis de mais fácil obtenção para estimar as demais 
com alto índice de precisão e exatidão. Uma dessas variáveis seria a propriedade de cor do material (BEKHTA \& NIEMZ, 2003; ESTEVES \& PEREIRA, 2009; GARCIA et al., 2014). Sabe-se que o escurecimento gradual da biomassa provocado pelo tratamento térmico está associado à oxidação e degradação parcial de alguns extrativos e componentes da parede celular (PINCELLI et al., 2012; ZANÚNCIO et al., 2014), que por sua vez tem correlação com a composição química e com o poder calorífico do material resultante.

Com isso, o objetivo do presente estudo foi avaliar as correlações existentes das propriedades colorimétricas com a composição química imediata e o poder calorífico de diferentes biomassas submetidas ao tratamento térmico e ajustar suas respectivas equações de regressão.

\section{MATERIAL E MÉTODOS}

Para a realização do experimento foram utilizadas três biomassas - serragem de eucalipto com casca (Eucalyptus sp.), bagaço de cana-de-açúcar (Saccharum sp.) e cavacos de bambu.

As biomassas foram secas em estufa a $103 \pm 2{ }^{\circ} \mathrm{C}$ com ci rculação de ar até atingir massa constante. Posteriormente, foram submetidas ao tratamento térmico dentro de um forno tipo mufla. As amostras ficaram acondicionadas em um container metálico dentro do forno com saída para os gases voláteis. Iniciou-se o processo a $100^{\circ} \mathrm{C}$, com taxa de aquecimento posterior de $1,6^{\circ} \mathrm{C} / \mathrm{min}$. Foram avaliadas quatro temperaturas finais, sendo $250,300,350$ e $400^{\circ} \mathrm{C}$. A pós o tratamento térmico, as amostras foram moídas e peneiras, recolhendo-se a fração que ficou retida na malha de 60 mesh.

Para a determinação dos teores de materiais voláteis (MV), carbono fixo (CF) e cinzas foi realizada a análise química imediata, de acordo com metodologia descrita pela norma ABNT NBR 8112 (ABNT, 1983). O Poder Calorífico Superior (PCS) foi determinado de acordo com a metodologia descrita pela norma da ABNT NBR 8633 (ABNT, 1984), utilizando-se uma bomba calorimétrica adiabática.

A análise colorimétrica foi realizada de acordo com o sistema CIE L*a*b* da norma da Commission Internationale de L'Eclairage. Esse sistema trabalha com três índices de cor: a claridade (L) que varia de zero (preto) a 100 (branco) e as coordenadas cromáticas do Matiz Vermelho $\left(a^{*}\right)$ e do Matiz Amarelo $\left(b^{*}\right)$, ambas variando entre -60 e +60 de acordo com suas respectivas purezas (GARCIA et al., 2014; ZANÚNCIO et al., 2014). Os índices foram obtidos com um espectrofotômetro portátil Konica Minolta CM-2500D, efetuando-se as medições por meio do contato direto e perpendicular a superfície das amostras.

O experimento foi analisado segundo um Delineamento Inteiramente Casualizado com 15 tratamentos ( 3 biomassa e 4 temperaturas, além das testemunhas) em três repetições. As correlações entre os índices colorimétricos, a composição química imediata e o poder calorífico, com suas respetivas equações de regressão, foram obtidas a 5\% de probabilidade utilizando o software Statistica 12.0. Quando encontrado resultados significativos na Análise de Regressão, foram ajustadas as equações utilizando os três índices colorimétricos, segundo o modelo:

$$
Y=\beta 0+L^{*} \beta 1+a^{*} \beta 2+b^{*} \beta 3+e
$$

Sendo $Y$ o valor estimado para a propriedade; $\beta 0, \beta 1$ e $\beta 2$ os coeficientes gerados; $L$ o valor de claridade; $a$ o valor de Matiz Vermelho; $b$ o valor de Matiz Amarelo; $e$ o erro de estimativa. 


\section{RESULTADOS E DISCUSSÃO}

$\mathrm{Na}$ Tabela 1 são apresentados os valores de média, desvio-padrão e coeficiente de variação dos índices colorimétricos (Claridade, Matiz Vermelho e Matiz Amarelo) das biomassas submetidas a tratamento térmico. Observa-se que os índices colorimétricos reduziram em função do aumento da temperatura máxima a qual a biomassa foi submetida.

TABELA 1 - Colorimetria de três biomassas submetidas ao processo de tratamento térmico em diferentes temperaturas. Média, Desvio-padrão (DP) e Coeficiente de variação (CV)

\begin{tabular}{|c|c|c|c|c|c|c|c|c|c|c|}
\hline \multirow[t]{2}{*}{ Material } & \multirow[t]{2}{*}{ Temp.( $\left.{ }^{(} \mathrm{C}\right)$} & \multicolumn{3}{|c|}{ Claridade (L) } & \multicolumn{3}{|c|}{ Matiz Vermelho $\left(a^{*}\right)$} & \multicolumn{3}{|c|}{ Matiz Amarelo $\left(b^{\star}\right)$} \\
\hline & & Média & DP & CV & Média & DP & CV & Média & $\mathrm{DP}$ & $\mathrm{CV}$ \\
\hline \multirow{5}{*}{$\begin{array}{c}\text { Serragem } \\
\text { de } \\
\text { Eucalipto }\end{array}$} & Test. & 53,83 & 0,60 & 1,12 & 16,87 & 0,32 & 1,91 & 20,63 & 0,36 & 1,48 \\
\hline & 250 & 19,23 & 0,93 & 4,83 & 4,07 & 0,06 & 1,42 & 3,13 & 0,21 & 6,64 \\
\hline & 300 & 18,17 & 0,49 & 2,72 & 0,73 & 0,21 & 28,39 & $-0,20$ & 0,10 & $-50,00$ \\
\hline & 350 & 18,87 & 0,98 & 5,20 & 0,50 & 0,60 & 120,00 & $-0,20$ & 0,10 & $-50,00$ \\
\hline & 400 & 20,77 & 0,21 & 1,00 & 0,37 & 0,15 & 41,66 & $-0,50$ & 0,10 & $-20,00$ \\
\hline \multirow{5}{*}{$\begin{array}{c}\text { Bagaço } \\
\text { de Cana- } \\
\text { de- } \\
\text { açúcar }\end{array}$} & Test. & 49,73 & 0,98 & 1,97 & 11,43 & 0,61 & 5,34 & 25,13 & 0,50 & 2,00 \\
\hline & 250 & 18,17 & 1,07 & 5,89 & 4,60 & 0,17 & 3,77 & 4,00 & 0,17 & 4,33 \\
\hline & 300 & 19,00 & 0,20 & 1,05 & 0,83 & 0,15 & 18,33 & $-0,23$ & 0,38 & $-162,25$ \\
\hline & 350 & 17,67 & 1,59 & 8,99 & 0,77 & 0,21 & 27,15 & $-0,43$ & 0,06 & $-13,32$ \\
\hline & 400 & 18,70 & 0,79 & 4,24 & 0,17 & 0,50 & 301,99 & $-0,43$ & 0,06 & $-13,32$ \\
\hline \multirow{5}{*}{ Bambu } & Test. & 63,50 & 0,50 & 0,79 & 11,47 & 0,57 & 4,96 & 28,53 & 0,67 & 2,33 \\
\hline & 250 & 18,03 & 0,23 & 1,28 & 1,63 & 0,15 & 9,35 & 1,30 & 0,10 & 7,69 \\
\hline & 300 & 18,60 & 0,72 & 3,88 & 1,03 & 0,49 & 47,74 & 0,70 & 0,30 & 42,86 \\
\hline & 350 & 18,40 & 0,56 & 3,03 & 0,97 & 0,12 & 11,95 & 0,63 & 0,29 & 45,58 \\
\hline & 400 & 19,27 & 0,51 & 2,66 & 1,30 & 1,05 & 81,04 & 0,07 & 0,21 & 312,25 \\
\hline
\end{tabular}

Os índices de claridade das três biomassas reduziram consideravelmente quando estas foram submetidas ao tratamento a $250^{\circ} \mathrm{C}$. A partir dessa temperatura os valores mantiveram-se praticamente estáveis, com variações mínimas entre si. 0 bambu foi a biomassa in natura que apresentou o maior índice de claridade, enquanto o bagaço de cana-de-açúcar apresentou o menor.

Os índices de Matiz Vermelho e de Matiz Amarelo também reduziram consideravelmente quando submetidos ao tratamento a $250^{\circ} \mathrm{C}$. Entre 250 e $300^{\circ} \mathrm{C}$, houve outra redução de menor amplitude. A partir dessa temperatura, os índices obtiveram variações mínimas, chegando a apresentar valores negativos de Matiz Amarelo para temperaturas mais elevadas. O bagaço de cana-de-açúcar e o bambu apresentaram valores mais altos do Matiz Amarelo do que a serragem de eucalipto que, por sua vez, apresentou o maior valor médio do Matiz Vermelho.

Conforme descrito por BEKHTA \& NIEMZ (2003), as alterações de cor são drásticas em tratamentos térmicos acima de $200{ }^{\circ} \mathrm{C}$, p rovocando um escurecimento gradual da biomassa. Fato confirmado neste estudo pela redução do valor $L$ da análise colorimétrica que registra o índice de claridade do material. ESTEVES et al. (2008) citam a degradação das hemiceluloses como sendo a causa mais relevante 
para o escurecimento da biomassa. MOURA \& BRITO (2011) citam que com o aumento da temperatura, a biomassa é convertida em carvão vegetal, convergindo para a cor preta, independentemente da espécie.

GARCIA et al. (2014) listaram uma combinação de fatores para explicar a mudança de cor da biomassa após o tratamento térmico, dentre os quais estão a degradação e oxidação dos compostos extrativos; formação de produtos oxidativos da degradação parcial dos componentes estruturais da parede celular; migração dos extrativos e outros compostos não estruturais do interior para a superfície do material; e aumento do teor de lignina com relação ao teor de polissacarídeos.

O Matiz Vermelho (a) e o Matiz Amarelo $(b)$ referem-se às purezas dessas respectivas tonalidades. Os extrativos são os principais componentes cromóforos que interferem nesses índices da análise de colorimetria (PINCELL et al., 2012). Dentre as biomassas utilizadas (Tabela 1), a serragem do eucalipto apresentou 0 maior valor de a e o bambu o maior valor de $b$, demonstrando que essas biomassas in natura apresentam maiores tonalidades de vermelho e de amarelo, respectivamente. A maioria dos extrativos responsáveis por essas tonalidades é volatizada em temperaturas inferiores a $250^{\circ} \mathrm{C}$, fato que corrobora, portanto, com a redução brusca dos índices $a$ e $b$ deste estudo (MOURA \& BRITO, 2011; CARVALHO et al., 2014; ZANÚNCIO et al., 2014).

$\mathrm{Na}$ Tabela 2 é apresentada a Matriz de Correlações entre os índices gerais de colorimetria (L, a e b), a composição química imediata (Materiais Voláteis, Carbono Fixo e Cinzas) e o Poder Calorífico Superior.

TABELA 2 - Matriz de correlações entre os índices de colorimetria, composição química e poder calorífico das biomassas termicamente tratadas

\begin{tabular}{cccccccc}
\hline Variável & $\mathrm{L}$ & $\mathrm{a}$ & $\mathrm{B}$ & $\mathrm{MV}$ & $\mathrm{CF}$ & Cinzas & PCS \\
\hline $\mathrm{L}$ & 1,000 & $0,915^{*}$ & $0,979^{*}$ & $0,839^{*}$ & $-0,829^{*}$ & $-0,384^{*}$ & $-0,781^{*}$ \\
A & $\ldots$ & 1,000 & $0,923^{*}$ & $0,912^{*}$ & $-0,902^{*}$ & $-0,410^{*}$ & $-0,874^{*}$ \\
B & $\ldots$ & $\ldots$ & 1,000 & $0,899^{*}$ & $-0,883^{*}$ & $-0,464^{*}$ & $-0,849^{*}$ \\
MV & $\ldots$ & $\ldots$ & $\ldots$ & 1,000 & $-0,994^{*}$ & $-0,408^{*}$ & $-0,972^{*}$ \\
CF & $\ldots$ & $\ldots$ & $\ldots$ & $\ldots$ & 1,000 & $0,306^{*}$ & $0,965^{*}$ \\
Cinzas & $\ldots$ & $\ldots$ & $\ldots$ & $\ldots$ & $\ldots$ & 1,000 & $0,411^{*}$ \\
PCS & $\ldots$ & $\ldots$ & $\ldots$ & $\ldots$ & $\ldots$ & $\ldots$ & 1,000 \\
\hline
\end{tabular}

A correlação significativa a $5 \%$ de probabilidade é identificada pelo asterisco $\left(^{*}\right)$. Sendo MV (Materiais Voláteis), CF (Carbono Fixo) e PCS. (Poder Calorífico Superior).

Verifica-se que houve interação significativa entre os índices colorimétricos, composição química imediata e PCS. Os índices de claridade (L), Matiz Vermelho (a) e Matiz Amarelo (b) apresentaram alta correlação positiva com os teores de materiais voláteis das amostras e alta correlação negativa com os teores de carbono fixo e PCS. As maiores correlações foram observadas entre os teores de materiais voláteis e os teores de carbono fixo e destes com o PCS. As interações com os teores de cinzas, apesar de estatisticamente significativas, não apresentaram correlações tão elevadas quanto às demais em decorrência das variações nos teores de cinzas terem sido numericamente baixas.

O tratamento térmico da biomassa provoca a eliminação parcial dos compostos mais instáveis na condição de materiais voláteis, principalmente das hemiceluloses e dos extrativos no caso da torrefação (ESTEVES \& PEREIRA, 2009; VAN DER STELT et al., 2011) e também da celulose no caso da carbonização ENCICLOPÉDIA BIOSFERA, Centro Científico Conhecer - Goiânia, v.13 n.23; p.1632 
(PEREIRA et al., 2013). Consequentemente, há um aumento gradual na concentração dos teores de lignina na fração sólida em função do aumento da temperatura. Diferentemente das hemiceluloses e das celuloses que são polissacarídeos com baixa relação $\mathrm{C} / \mathrm{O}$, a lignina é um composto aromático com altos teores de carbono, o que influencia diretamente no seu maior teor de carbono fixo (VITAL et al., 2013).

A biomassa na condição in natura apresenta teores de materiais voláteis três a quatro vezes superiores aos teores de carbono fixo. Quando submetido ao processo de carbonização, a proporção entre esses dois teores chega a inverter na constituição química com relação aos valores iniciais (OLIVEIRA et al., 2010). O aumento nos teores de carbono tem influência direta e positiva no potencial energético do combustível. Conforme citado JENKINS et al. (1998), o aumento em $1 \%$ na concentração de carbono de uma biomassa, pode elevar seu poder calorífico em até $93 \mathrm{kcal} / \mathrm{kg}$. A correlação entre índices de colorimetria com a composição química e o poder calorífico (Tabela 2), portanto, é explicada pelo fato dessas propriedades serem alteradas simultaneamente no decorrer na degradação térmica.

$\mathrm{Na}$ Tabela 3 são apresentadas as equações ajustadas e os seus respectivos coeficientes de determinação $\left(\mathrm{R}^{2}\right)$ obtidos para os Materiais Voláteis, Carbono Fixo, Cinzas e Poder Calorífico Superior. O teste F da Análise da Variância foi significativo para todas as correlações.

TABELA 3 - Equações de regressão ajustadas a partir das propriedades colorimétricas e seus respectivos coeficientes de determinação e erro de estimativa para biomassas tratadas termicamente

\begin{tabular}{|c|c|c|c|c|}
\hline Material & Variável & Equação de Regressão Ajustada & $\mathrm{R}^{2}$ & Erro \\
\hline \multirow{4}{*}{$\begin{array}{c}\text { Serragem } \\
\text { de } \\
\text { Eucalipto }\end{array}$} & MV & $97,68-3,2^{*} L+2,95^{*} a+5,22^{*} b$ & 0,98 & 3,69 \\
\hline & $\mathrm{CF}$ & $0,81+2,84^{*} L-2,46^{*} a-4,78^{*} b$ & 0,97 & 3,34 \\
\hline & Cinzas & $1,51+0,36^{*} L-0,5^{\star} a-0,44^{*} b$ & 0,98 & 0,38 \\
\hline & PCS & $4354,3+86,5^{\star} L-44,3^{*} a-185,1^{*} b$ & 0,97 & 134,4 \\
\hline \multirow{4}{*}{$\begin{array}{l}\text { Bagaço } \\
\text { de Cana- } \\
\text { de-açúcar }\end{array}$} & MV & $35,39-0,38^{*} L+7,59^{*} a-0,74^{*} b$ & 0,97 & 4,38 \\
\hline & CF & $62,33+0,36^{*} L-7,42^{*} a+0,75^{*} b$ & 0,97 & 4,27 \\
\hline & Cinzas & $2,27+0,02^{*} L-0,17^{*} a-0,01^{*} b$ & 0,96 & 0,14 \\
\hline & PCS & $6428,8-6,2^{*} L-402,9^{*} a+108,4^{*} b$ & 0,92 & 267,4 \\
\hline \multirow{4}{*}{ Bambu } & MV & $97,31-3,59^{*} L-1,89 * a+8,31^{*} b$ & 0,98 & 3,29 \\
\hline & CF & $2,58+3,42^{*} L+1,83^{*} a-7,94^{*} b$ & 0,98 & 3,14 \\
\hline & Cinzas & $0,11+0,17^{*} L+0,06^{*} a-0,37^{*} b$ & 0,97 & 0,15 \\
\hline & PCS & $4340,9+113^{*} \mathrm{~L}+47,1^{*} \mathrm{a}-261,7^{*} \mathrm{~b}$ & 0,97 & 140,3 \\
\hline
\end{tabular}

Verifica-se que as equações de regressão geradas para estimar a composição química e o poder calorífico da biomassa termicamente tratada a partir dos índices de cor apresentaram altos coeficientes de determinação, todos acima de 0,9 . Os erros de estimativa foram maiores para as equações de estimativa do PCS e menor para os teores de cinzas. Os menores valores de $R^{2}$ e maiores erros de estimativas das equações ajustadas para o PCS estão relacionados aos valores numéricos naturalmente maiores desse índice de qualidade.

$\mathrm{Na}$ Figura 1 são apresentados os gráficos de dispersão dos dados obtidos para a composição química imediata e o poder calorífico superior, seguindo as 
normas ABNT, de cada biomassa em função da temperatura e as suas respectivas curvas estimadas pelas equações de regressão ajustadas a partir das variáveis colorimétricas.

Serragem de Eucalipto

M.V.

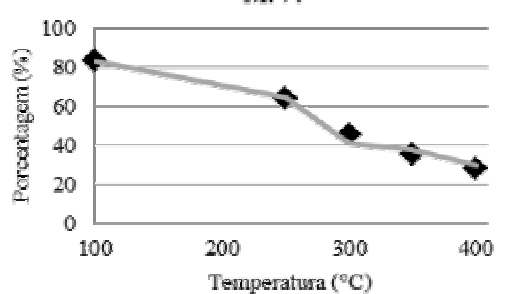

C.F.

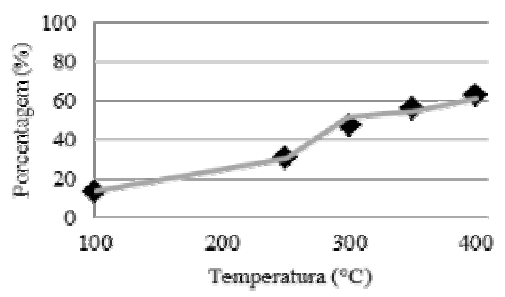

Cinzas

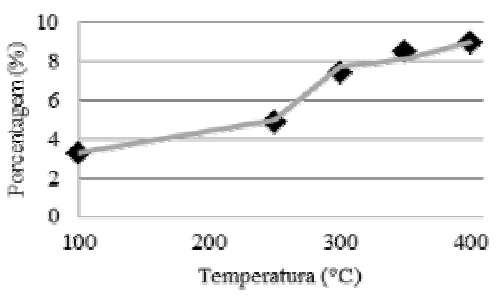

P.C.S.

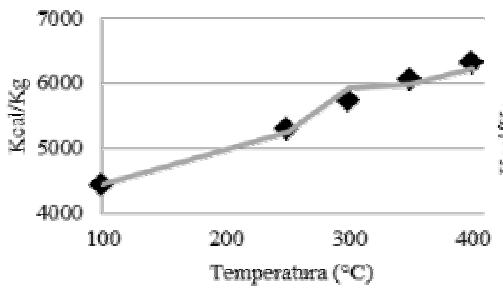

Bagaço de Cana-de-açúcar

M.V.

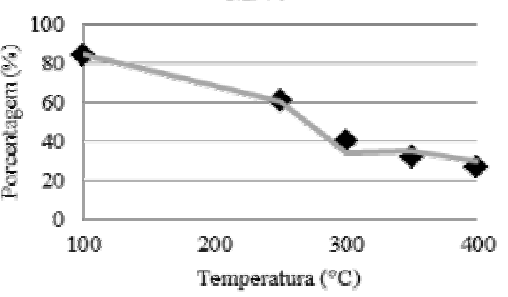

C.F.

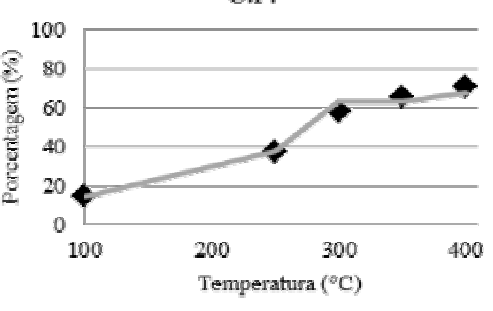

Cinzas

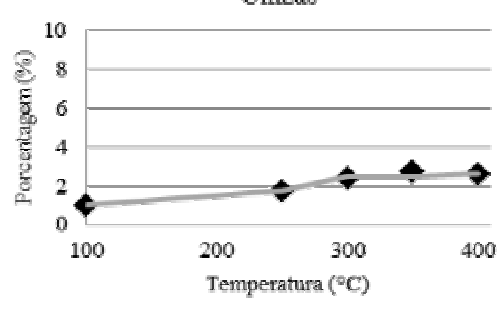

P.C.S.

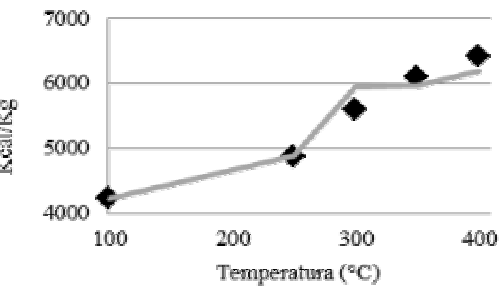

Bambu

M.V,

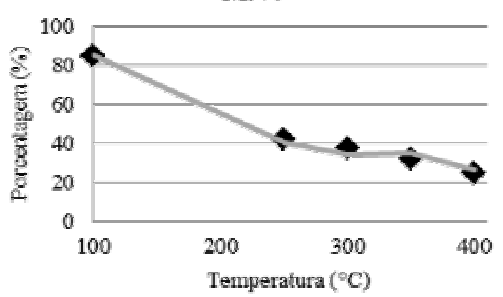

C.F.

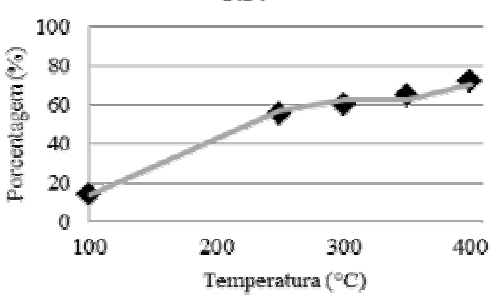

Cinzas

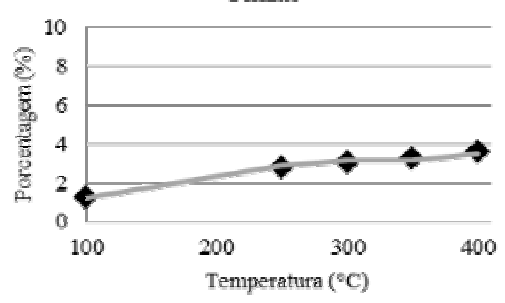

P.C.S.

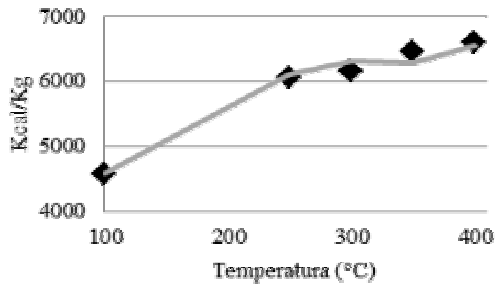

FIGURA 1 - Dispersão dos dados obtidos para as propriedades de cada biomassa em função da temperatura e as suas respectivas curvas estimadas pelas equações de regressão ajustadas a partir das variáveis colorimétricas.

Os gráficos mostram que existe uma correlação satisfatória entre os valores obtidos através das análises normatizadas e os valores estimados através das equações de regressão envolvendo os índices de cor. O Poder Calorífico Superior foi a variável que apresentou o ajuste menos adequado, principalmente para 0 bagaço de cana-de-açúcar que apresentou o menor $\mathrm{R}^{2}$.

As equações ajustadas apresentaram coeficientes de determinação acima de 0,9 , o que é satisfatório para estimar propriedades com muitas variáveis influenciáveis. Os erros de estimativas e os gráficos de dispersão (Figura 1) demonstram que as equações geradas apresentam ajustes adequados para a maioria das propriedades avaliadas. A indústria busca uma estimativa rápida e barata dessas propriedades, não exigindo um grau de precisão e exatidão tão 
elevado, assim, a metodologia proposta e avaliada é viável e tem potencial de aplicação no setor.

Ressalta-se que é necessário o ajuste de equações para cada situação específica, considerando a biomassa utilizada e a faixa de temperatura em que são aplicados os tratamentos térmicos. A metodologia torna-se menos eficiente para biomassas tratadas em temperaturas muito elevadas, pois os índices calorimétricos tendem a apresentar variações cada vez menos significativas em função do acréscimo de temperatura.

\section{CONCLUSÕES}

Os índices colorimétricos seguindo o sistema CIE $\mathrm{L}^{*} \mathrm{a}^{*} \mathrm{~b}^{*}$, a composição química imediata e o poder calorífico superior de biomassas termicamente tratadas apresentam tendência de variação em função da temperatura a que foram submetidas durante os tratamentos. Consequentemente, esses índices de qualidade apresentam correlações significativas entre si, possibilitando o ajuste de equação a partir dos índices colorimétricos com coeficientes de determinação altamente satisfatórios para a estimação das propriedades avaliadas. A metodologia apresentada, portanto, é uma ferramenta adequada para atender a demanda do setor industrial por análises alternativas mais rápidas do que as tradicionais. Porém, vale ressaltar que a metodologia não atingiu o grau de precisão e exatidão geral exigido pelas normas de pesquisa e que essa ferramenta só é viável com ajuste de equações adequado para cada biomassa específica.

\section{AGRADECIMENTOS}

À CAPES e à Embrapa Florestas pela concessão de bolsa e apoio ao projeto.

\section{REFERÊNCIAS}

ARIAS, B.; PEVIDA, C.; FERMOSO, J.; PLAZA, M. G.; RUBIERA, F.; PIS, J. J. Influence of torrefaction on the grindability and reactivity of woody biomass. Fuel Processing Technology, v.89, p.169-175, 2008.

ASSOCIAÇÃO BRASILEIRA DE NORMAS TÉCNICAS - ABNT. NBR 8112: Carvão Vegetal - Análise Imediata. Rio de Janeiro. 1983.

ASSOCIAÇÃO BRASILEIRA DE NORMAS TÉCNICAS - ABNT. NBR 8633: Carvão Vegetal: Determinação do Poder Calorífico. Rio de Janeiro. 1984.

BEKHTA, P.; NIEMZ, P. Effect of High Temperature on the Change in Color, Dimensional Stability and Mechanical Properties of Spruce Wood. Holzforschung, v.57, p.539-546, 2003.

CARVALHO, A.G.; ZANÚNCIO, A. J. V.; VITAL, B. R.; CARNEIRO, A. C. O.; SILVA, C. M. S. Colorimetric and Chemical Changes in Pre-Hydrolyzed Strand Board Particles of Pine and Eucalyptus. BioResources, v.9, n.4, p.7234-7242, 2014.

ESTEVES, B.; MARQUES, A. V.; DOMINGOS, I.; PEREIRA, H. Heat-induced colour changes of pine (Pinus pinaster) and eucalypt (Eucalyptus globulus) wood. Wood Science and Technology, v.42, n.5, p.369-384, 2008. 
ESTEVES, B. M.; PEREIRA, H. M. Wood modification by heat treatment: a review. BioResources, v.4, n.1, p.370-404, 2009.

GARCIA, R.A.; LOPES, J.O.; SANTOS, W.A. Modificação da cor original da madeira de Eucalyptus grandis através de tratamentos termorretificadores. Cerne, v.20, n.3, p. 449-457, 2014.

JENKINS, B. M.; BAXTER, L. L.; JUNIOR, T. R. M.; MILES, T. R.; Combustion properties of biomass. Fuel Processing Technology, v.54, p.17-46, 1998.

MOURA, L. F.; BRITO, J. O. Efeito da termorretificação sobre as propriedades colorimétricas das madeiras de Eucalyptus grandis e Pinus caribaea var. hondurensis. Scientia Forestalis, v.39, n.89, p.69-76, 2011.

OLIVEIRA, A.C.; CARNEIRO, A. C. O.; VITAL, B. R.; ALMEIDA, W.; PEREIRA, B. L. C.; CARDOSO, M. T. Parâmetros de qualidade da madeira e do carvão vegetal de Eucalyptus pellita F. Muell. Scientia Forestalis, v.38, n.87, p.431-439, 2010.

OLIVEIRA, A.C.; CARNEIRO, A. C. O.; PEREIRA, B. L. C.; VITAL, B. R.; CARVALHO, A. M. M. L.; TRUGILHO, P. F.; DAMÁSIO, R. A. P. Otimização da produção do carvão vegetal por meio do controle de temperaturas de carbonização. Revista Árvore, v.37, n.3, p.557-566, 2013.

PEREIRA, B. L. C.; CARNEIRO, A. C. O.; CARVALHO, A. M. M. L.; COLODETTE, J. L.; OLIVEIRA, A. C.; FONTES, M. P. F. Influence of chemical composition of eucalyptus wood on gravimetric yield and charcoal properties. Bioresources, v.8, n.3, p.4574-4592, 2013.

PINCELLI, A. L. P. S. M.; MOURA, L. F.; BRITO, J. O. Effect of thermal rectification on colors of Eucalyptus saligna and Pinus caribaea woods. Maderas. Ciencia y Tecnología, v.14, n.2, p.239-248, 2012.

VAN DER STELT, M. J. C.; GERHAUSER, H.; KIEL, J. H. A.; PTASINSKI, K. J. Biomass upgrading by torrefaction for the production of biofuels: A review. Biomass and Bioenergy, v.35, p.3748-3762, 2011.

VITAL, B.R.; CARNEIRO, A.C.O.; PEREIRA, B.L.C. Qualidade da madeira para fins energéticos. In: Bioenergia \& Biorrefinaria - Cana-de-açúcar \& Espécies Florestais. Editores: SANTOS, F.; COLODETTE, J.; QUEIROZ, J.H. Viçosa, MG. 2013. Pág $321-354$.

ZANUNCIO, A. J. V.; NOBRE, J. R. C.; MOTTA, J. P.; TRUGILHO, P. F. Química e Colorimetria da madeira de Eucalyptus grandis W. Mill ex Maiden termorretificada. Revista Árvore, v.38, n.4, p.765-770, 2014. 